\title{
Editorial \\ Special Issue on Application of Electronic Devices on Intelligent System
}

\author{
Teen-Hang Meen ${ }^{1, *(1)}$ and Cheng-Chien Kuo ${ }^{2}$ (i) \\ 1 Department of Electronic Engineering, National Formosa University, Yunlin 632, Taiwan \\ 2 Department of Electrical Engineering, National Taiwan University of Science and Technology, \\ Taipei 106, Taiwan; cckuo@mail.ntust.edu.tw \\ * Correspondence: thmeen@gs.nfu.edu.tw
}

check for updates

Citation: Meen, T.-H.; Kuo, C.-C. Special Issue on Application of Electronic Devices on Intelligent System. Electronics 2021, 10, 1506. https://doi.org/10.3390/ electronics10131506

Received: 16 June 2021

Accepted: 18 June 2021

Published: 22 June 2021

Publisher's Note: MDPI stays neutral with regard to jurisdictional claims in published maps and institutional affiliations.

Copyright: (C) 2021 by the authors. Licensee MDPI, Basel, Switzerland. This article is an open access article distributed under the terms and conditions of the Creative Commons Attribution (CC BY) license (https:// creativecommons.org/licenses/by/ $4.0 /)$.

\section{Introduction}

In a modern technological society, electronic engineering and design innovations are both academic and practical engineering fields that involve systematic technological materialization through scientific principles and engineering designs. Engineers and designers must work together with a variety of other professionals in their quest to find systems solutions to complex problems. Fast advances in science and technology have broadened the horizons of engineering whilst simultaneously creating a multitude of challenging problems in every aspect of modern life. Current research is interdisciplinary in nature, reflecting a combination of concepts and methods that often span several areas of mechanics, mathematics, electrical engineering, control engineering, and other scientific disciplines. The International Institute of Knowledge Innovation and Invention (IIKII, http: / /www.iikii.org) is an institute that promotes the exchange of innovations and inventions and establishes a communication platform for international innovations and research. In 2020, IIKII cooperated with the IEEE Tainan Section Sensors Council to hold three IEEE conferences: IEEE ECBIOS 2020, IEEE ICKII 2020, and IEEE ECICE 2020. This Special Issue titled "Application of Electronic Devices on Intelligent System" selects 14 excellent papers form 700 papers presented in these three IEEE conferences about the fields of electronic devices on intelligent system. The topics include as follows: electrical circuits and devices, microelectronics and computer technology, computer science and engineering, communications and information processing, electrical engineering communications. The main goals of this Special Issue are to encourage scientists to publish their experimental and theoretical results in as much detail as possible, and to discover new scientific knowledge relevant to the topics of electronics.

\section{The Topics of Intelligent Electronic Devices and Its Applications}

This Special Issue on "Intelligent Electronic Devices" selected 14 excellent papers from 700 papers presented in three IEEE conferences in 2020 on the topics of electronics. The published papers are introduced as follows:

Tseng et al. reported "Buck-Boost/Flyback Hybrid Converter for Solar Power System Applications" [1]. In this paper, the authors propose a hybrid converter to supply power from solar power source to load. Since power is generated by solar power, which depends on the intensity of solar power, the power generated by the solar power does not keep at a constant power. Experimental results which are obtained from a prototype with output voltage of $10 \mathrm{~V}$ and maximum output power $20 \mathrm{~W}$ have been implemented to verify its feasibility. It is suitable for an electronic sign indicating LED within $200 \mathrm{~W}$, which is used in the night time.

Huang et al. reported "Planning and Research of Distribution Feeder Automation with Decentralized Power Supply" [2]. The high penetration of distributed generation in distributed energy systems causes the variation of power loss and makes the power 
grid become more complicated, so this paper takes various types of optimal algorithms into account and simulates the feeder reconfiguration on the IEEE-33 system as well as the Taiwan power system. In the IEEE-33 system, the authors achieved a 52.57\% power loss reduction after feeder reconfiguration, and a $70.55 \%$ power loss reduction after the distributed generator was implemented and feeder reconfiguration. Under the variation of load demand and power generation of the Taiwan power system, the authors establish the system models by forecasting one-day load demand, and propose a one-day feeder switch operation strategy by considering the switches' operation frequency with the reduction of $83.3 \%$ manual operation and recommend feeder automation to achieve feeder power loss reduction, voltage profile improvement and get regional power grid resilient configuration.

Victor et al. reported "Fuzzy ARTMAP-Based Fast Object Recognition for Robots Using FPGA" [3]. This article shows the design and implementation of an invariant recognition machine vision system to compute a descriptive vector called the Boundary Object Function (BOF) using the Fuzzy ARTMAP (FAM) Neural Network. The object recognition machine is integrated in the Zybo Z7-20 module that includes reconfigurable FPGA hardware and a RISC processor. Object encoding, description and prediction is carried out rapidly compared to the processing time devoted to video capture at the camera's frame rate. Benefiting from parallel computing, the authors calculated the object's centroid and boundary points while acquiring the progressive image frame; all that was done with the intention of readying it for neural processing. The remaining time was devoted to recognizing the object, this caused low latency $(1.47 \mathrm{~ms})$. The test-bed also included TCP/IP communication to send/receive part location for grasping operations with an industrial robot to evaluate the approach. Results demonstrate that the hardware integration of the video sensor, image processing, descriptor generator, and the ANN classifier for cognitive decision on a single chip can increase the speed and performance of intelligent robots designed for smart manufacturing.

Tseng et al. reported "Bridgeless Boost Converter with an Interleaving Manner for PFC Applications" [4]. This paper proposes a bridgeless boost converter to increase the power factor of power systems using a utility line source for raising power quality. To reduce input and output current ripple, an interleaving manner is adopted in the proposed power system. When the interleaving bridgeless boost converter is used to implement power factor correction (PFC), it needs two bridgeless boost converters to process power during one switching cycle. In order to simplify the proposed bridgeless boost converter, two sets of switches in the conventional bridgeless boost one are integrated to reduce component counts. With this approach, the proposed bridgeless boost converter uses four switches to implement PFC features. Therefore, the proposed boost converter can increase conversion efficiency and decrease component counts, resulting in a higher conversion efficiency, lower cost and more simplicity for driving circuits. Finally, a prototype with a universal input voltage source (AC $90 \mathrm{~V} 265 \mathrm{~V}$ ) under an output voltage of $400 \mathrm{~V}$ and a maximum output power of $1 \mathrm{~kW}$ has been implemented to verify the feasibility of the proposed bridgeless boost converter.

Chen et al. reported "Lower-Limb Electromyography Signal Analysis of Distinct Muscle Fitness Norms under Graded Exercise Intensity" [5]. The aim of this study was to analyze the correlation between muscle fitness and the electromyography (EMG) signals of lower limbs under varying exercise intensity. The standing long jump was used as a test task for assessing the power of the lower limb muscles. Participants were university freshmen who belonged to the top $20 \%$, middle $20 \%$, and bottom $20 \%$ groups in terms of physical fitness norms. The EMG signals of the participants' lower limbs while they performed squats were collected under four exercise intensities of repetitions maximum (RM): no load, $8 \mathrm{RM}, 18 \mathrm{RM}$, and 28RM; the features of the signals were extracted using time-domain and frequency-domain analysis. Statistical analysis was also performed. The top and bottom groups exhibited significant differences time-domain indicators mean absolute value (MAV) and average amplitude change (AAC) in the low-intensity exercise (28RM). The MAV, variance of EMG (VAR), root mean square (RMS), and AAC were 
significantly different between the top and bottom groups in the three graded intensities (8RM, 18RM, and 28RM). The mean frequency (MNF) and median frequency (MDF), which are frequency-domain indicators, were significantly different between the top and bottom groups in the low-intensity (28RM) and moderate-intensity (18RM) exercises.

Chen et al. reported "Hot Carrier Stress Sensing Bulk Current for $28 \mathrm{~nm}$ Stacked High-k nMOSFETs" [6]. This work primarily focuses on the degradation degree of bulk current (IB) for $28 \mathrm{~nm}$ stacked high-k (HK) n-channel metal-oxide-semiconductor fieldeffect transistors (MOSFETs), sensed and stressed with the channel-hot-carrier test and the drain-avalanche-hot-carrier test, and uses a lifetime model to extract the lifetime of the tested devices. The results show that when IB reaches its maximum, the ratio of VGS/VDS values at this point, in the meanwhile, gradually increases in the tested devices from the long-channel to the short ones, not just located at one third to one half. The possible ratiocination is due to the ON-current (IDS), in which the short-channel devices provide larger IDS impacting the drain junction and generating more hole carriers at the surface channel near the drain site. In addition, the decrease in IB after hot-carrier stress is not only the increment in threshold voltage VT inducing the decrease in IDS, but also the increment in the recombination rate due to the mechanism of diffusion current. Ultimately, the device lifetime uses Berkley's model to extract the slope parameter $\mathrm{m}$ of the lifetime model. Previous studies have reported m-values ranging from 2.9 to 3.3, but in this case, approximately 1.1 . This possibly means that the critical energy of the generated interface state becomes smaller, as is the barrier height of the HK dielectric to the conventional silicon dioxide as the gate oxide.

Chen et al. reported "Q-Factor Performance of $28 \mathrm{~nm}$-Node High-K Gate Dielectric under DPN Treatment at Different Annealing Temperatures" [7]. Q-factor is a reasonable index to investigate the integrity of circuits or devices in terms of their energy or charge storage capabilities. The authors use this figure of merit to explore the deposition quality of nano-node high-k gate dielectrics by decoupled-plasma nitridation at different temperatures with a fixed nitrogen concentration. This is very important in radio-frequency applications. From the point of view of the Q-factor, the device treated at a higher annealing temperature clearly demonstrates a better $Q$-factor value. Another interesting observation is the appearance of two troughs in the Q-VGS characteristics, which are strongly related to either the series parasitic capacitance, the tunneling effect, or both.

Alotaibi reported "Automated and Intelligent System for Monitoring Swimming Pool Safety Based on the IoT and Transfer Learning" [8]. This paper proposes an efficient and reliable detection system that utilizes a single image to detect and classify drowning objects, to prevent drowning incidents. The proposed system utilizes the IoT and transfer learning to provide an intelligent and automated solution for off-time monitoring swimming pool safety. In addition, a specialized transfer-learning-based model utilizing a model pretrained on "ImageNet", which can extract the most useful and complex features of the captured image to differentiate between humans, animals, and other objects, has been proposed. The proposed system aims to reduce human intervention by processing and sending the classification results to the owner's mobile device. The performance of the specialized model is evaluated by using a prototype experiment that achieves higher accuracy, sensitivity, and precision, as compared to other deep learning algorithms.

Wang et al. reported "A Non-Dissipative Equalizer with Fast Energy Transfer Based on Adaptive Balancing Current Control" [9]. In this study, an active inductive equalizer with fast energy transfer based on adaptive balancing current control is proposed to rapidly equilibrate lithium-ion battery packs. A multiphase structure of equalizer formed by many specific parallel converter legs (PCLs) with bidirectional energy conversion serves as the power transfer stage to make the charge shuttle back and forth between the cell and subpack or sub-pack and sub-pack more flexible and efficient. This article focuses on dealing with the problem of slow balancing rate, which inherently arises from the reduction in balancing current as the voltage difference between the cells or sub-packs decreases, especially in the later period of equalization. An adaptive varied-duty-cycle (AVDC) algorithm 
is put forward here to accelerate the balance process. The devised method has taken the battery nonlinear behavior and the nonideality of circuit component into consideration and can adaptively modulate the duty cycle with the change of voltage differences to maintain balancing current nearly constant in the whole equilibrating procedure. Test results derived from simulations and experiments are provided to demonstrate the validity and effectiveness of the equalizer prototype constructed. Comparing with the conventional fixed duty cycle (FDC) method, the improvements of $68.3 \%$ and $8.3 \%$ in terms of balance time and efficiency have been achieved.

Piedad et al. reported "Frequency Occurrence Plot-Based Convolutional Neural Network for Motor Fault Diagnosis" [10]. In this study, a healthy motor and four identical motors with synthetically applied fault conditions-bearing axis deviation, stator coil inter-turn short circuiting, a broken rotor strip, and outer bearing ring damage-are tested. A set of 150 three-second sampling stator current signals from each motor fault condition are taken under five artificial coupling loads $(0,25 \%, 50 \%, 75 \%$ and $100 \%)$. The sampling signals are collected and processed into frequency occurrence plots (FOPs) which later serve as CNN inputs. This is done first by transforming the time series signals into its frequency spectra then convert these into two-dimensional FOPs. Five-fold stratified sampling cross-validation is performed. When motor load variations are considered as input labels, FOP-CNN predicts motor fault conditions with a $92.37 \%$ classification accuracy. It precisely classifies and recalls bearing axis deviation fault and healthy conditions with $99.92 \%$ and $96.13 \% \mathrm{f}$-scores, respectively. When motor loading variations are not used as input data labels, FOP-CNN still satisfactorily predicts motor condition with an $80.25 \%$ overall accuracy. FOP-CNN serves as a new feature extraction technique for time series input signals such as vibration sensors, thermocouples, and acoustics.

Zhang et al. reported "FPGA Acceleration of CNNs-Based Malware Traffic Classification" [11]. In this study, convolutional neural networks (CNNs)-based malware traffic classification can automatically learn features from raw traffic, avoiding the inaccuracy of hand-design traffic features. Through the experiments and comparisons of LeNet, AlexNet, VGGNet, and ResNet, it is found that LeNet has good and stable classification ability for malware traffic and normal traffic. Then, a field programmable gate array (FPGA) accelerator for CNNs-based malware traffic classification is designed, which consists of a parameterized hardware accelerator and a fully automatic software framework. By fully exploring the parallelism between CNN layers, parallel computation and pipeline optimization are used in the hardware design to achieve high performance. Simultaneously, runtime reconfigurability is implemented by using a global register list. By encapsulating the underlying driver, a three-layer software framework is provided for users to deploy their pre-trained models. Finally, a typical CNNs-based malware traffic classification model was selected to test and verify the hardware accelerator. The typical application system can classify each traffic image from the test dataset in $18.97 \mu$ s with the accuracy of $99.77 \%$, and the throughput of the system is $411.83 \mathrm{Mbps}$.

Xiao et al. reported "Posit Arithmetic Hardware Implementations with The Minimum Cost Divider and SquareRoot" [12]. In this paper, the authors propose several hardware implementations that contain the Posit adder/subtractor, multiplier, divider, and square root. The goal is to achieve an arbitrary Posit format and exploit the minimum circuit area, which is required in embedded devices. To implement the minimum circuit area for the divider and square root, the alternating addition and subtraction method is used rather than the Newton-Raphson method. Compared with other works, the area of the divider is about $0.2 \times-0.7 \times($ FPGA). Furthermore, this paper provides the synthesis results for each critical module with the Xilinx Virtex-7 FPGA VC709 platform.

Lin et al. reported "Lung Nodule Classification Using Taguchi-Based Convolutional Neural Networks for Computer Tomography Images" [13]. In this study, a Taguchi-based convolutional neural network (CNN) was proposed for classifying nodules into malignant or benign. For setting parameters in a CNN, most users adopt trial and error to determine structural parameters. This study used the Taguchi method for selecting preliminary 
factors. The orthogonal table design is used in the Taguchi method. The final optimal parameter combination was determined, as were the most significant parameters. To verify the proposed method, the lung image database consortium data set from the National Cancer Institute was used for analysis. The database contains a total of 16,471 images, including 11,139 malignant nodule images. The experimental results demonstrated that the proposed method with the optimal parameter combination obtained an accuracy of $99.6 \%$.

Lam et al. reported "A Segmentation Enhancement Method for the Low-Contrast and Narrow-Banded Substances in CBCT Images" [14]. In this study, a new segmentation enhancement method for low-contrast and narrow-banded substances is proposed based on previous work on selective anatomy analysis iterative reconstruction (SA2IR). The purpose of the proposed method is to segment facial skin tissue based on combinatorial optimization and previously known facial soft tissue structure anatomy. The results using this method indicated that the skin thickness was much more easily and more quickly identified than with conventional ultrasonic scanning methods. This method holds the potential to be an assisting tool for studying linage of anthropometrics, forensics, human archaeology, and some narrow medico-dental applications.

Author Contributions: Writing and reviewing all papers, T.-H.M.; Checking and correcting the manuscript, C.-C.K. All authors have read and agreed to the published version of the manuscript.

Funding: This research received no external funding.

Acknowledgments: The Guest Editors would like to thank the authors for their contributions to this Special Issue and all the reviewers for their constructive reviews.

Conflicts of Interest: The authors declare no conflict of interest.

\section{References}

1. Tseng, S.Y.; Fan, J.H. Buck-Boost/Flyback Hybrid Converter for Solar Power System Applications. Electronics 2021, $10,414$. [CrossRef]

2. Huang, Y.C.; Chang, W.C.; Hsu, H.; Kuo, C.C. Planning and Research of Distribution Feeder Automation with Decentralized Power Supply. Electronics 2021, 10, 362. [CrossRef]

3. Lomas-Barrie, V.; Pena-Cabrera, M.; Lopez-Juarez, I.; Navarro-Gonzalez, J.L. Fuzzy ARTMAP-Based Fast Object Recognition for Robots Using FPGA. Electronics 2021, 10, 361. [CrossRef]

4. Tseng, S.Y.; Fan, J.H. Bridgeless Boost Converter with an Interleaving Manner for PFC Applications. Electronics 2021, 10, 296. [CrossRef]

5. Chen, C.K.; Lin, S.L.; Wang, T.C.; Lin, Y.J.; Wu, C.L. Lower-Limb Electromyography Signal Analysis of Distinct Muscle Fitness Norms under Graded Exercise Intensity. Electronics 2020, 9, 2147. [CrossRef]

6. Chen, C.W.; Wang, M.C.; Chang, C.H.T.; Chu, W.L.; Sung, S.P.; Lan, W.H. Hot Carrier Stress Sensing Bulk Current for 28 nm Stacked High-k nMOSFETs. Electronics 2020, 9, 2095. [CrossRef]

7. Chen, C.W.; Wang, S.J.; Hsieh, W.C.; Chen, J.M.; Jong, T.; Lan, W.H.; Wang, M.C. Q-Factor Performance of 28 nm-Node High-K Gate Dielectric under DPN Treatment at Different Annealing Temperatures. Electronics 2020, 9, 2086. [CrossRef]

8. Alotaibi, A. Automated and Intelligent System for Monitoring Swimming Pool Safety Based on the IoT and Transfer Learning. Electronics 2020, 9, 2082. [CrossRef]

9. Wang, S.C.; Liu, C.Y.; Liu, Y.H. A Non-Dissipative Equalizer with Fast Energy Transfer Based on Adaptive Balancing Current Control. Electronics 2020, 9, 1990. [CrossRef]

10. Piedad, E.J.; Chen, Y.T.; Chang, H.C.; Kuo, C.C. Frequency Occurrence Plot-Based Convolutional Neural Network for Motor Fault Diagnosis. Electronics 2020, 9, 1711. [CrossRef]

11. Zhang, L.; Li, B.; Liu, Y.; Zhao, X.; Wang, Y.; Wu, J. FPGA Acceleration of CNNs-Based Malware Traffic Classification. Electronics 2020, 9, 1631. [CrossRef]

12. Xiao, F.; Liang, F.; Wu, B.; Liang, J.; Cheng, S.; Zhang, G. Posit Arithmetic Hardware Implementations with the Minimum Cost Divider and SquareRoot. Electronics 2020, 9, 1622. [CrossRef]

13. Lin, C.J.; Li, Y.C. Lung Nodule Classification Using Taguchi-Based Convolutional Neural Networks for Computer Tomography Images. Electronics 2020, 9, 1066. [CrossRef]

14. Lam, D.N.; Liu, C.F.; Du, Y.C. A Segmentation Enhancement Method for the Low-Contrast and Narrow-Banded Substances in CBCT Images. Electronics 2020, 9, 974. 